\title{
The rational points close to a curve III
}

\author{
by \\ M. N. HuXley (Cardiff)
}

1. Introduction. If a function $f(x)$ takes values which are approximately rational at integer points or at rational points, is $f(x)$ approximately a rational function? This is a badly posed question, best answered in the contrary sense, and made quantitative. So we want to show that if $f(x)$ cannot be approximated over a long subinterval of $[0, M]$ by a rational function $u(x) / v(x)$, where $u(x)$ and $v(x)$ are polynomials of degree at most $d$, then

$$
\left|f(m)-\frac{r}{q}\right| \leq \delta
$$

does not have a solution $r / q$ with $1 \leq q \leq Q$ for most integers $m=$ $0,1, \ldots, M$, when $\delta$ is sufficiently small; and if $F(x)$ cannot be approximated over a long subinterval of $[0,1]$ by $u(x) / v(x)$ as above, then

$$
\left|F\left(\frac{m}{n}\right)-\frac{r}{q}\right| \leq \delta
$$

does not have a solution $r / q$ with $1 \leq q \leq Q$ for most rational numbers $m / n$ with $n=1, \ldots, M, m=0, \ldots, n$, and $(m, n)=1$. A good quantitative bound would find some applications in classical number theory. For example, if there are many gaps of length at least $H$ between $k$-free numbers, then the inequality

$$
m^{k} q-n^{k} r=h, \quad 1 \leq h \leq H,
$$

has many solutions with $m$ and $n$ large ([4]). We see that (1.3) implies an inequality of the form (1.2).

The integers on or close to a smooth curve have been well studied (see $[8],[9],[7],[1]$, and the book [3] for further references). In [2] we considered

$$
\left|f\left(\frac{m}{q}\right)-\frac{n}{q}\right| \leq \delta
$$

2000 Mathematics Subject Classification: 11J25, 11P21, 11K38. 
regarding $(m, n, q)$ as a point in the projective plane, and using duality between the points of a curve and its tangent lines. In (1.1) or (1.2) we approximate $f(x)$ or $F(x)$ by a ratio $u(x) / v(x)$ of polynomials of degree at most $d$. In [5] we took $d=1$ and used a geometric invariant, the crossratio. In this paper $d$ is a fixed positive integer. The test for the trivial case $f(x) \equiv u(x) / v(x)$ is that a determinant of order $2 d+2$ should vanish identically. The rational numbers $r / q$ are function values up to an accuracy $\delta$, but the determinant is unstable under perturbation, and our results (in the case when $f(x)$ is bounded) become non-trivial when $\delta=1 / Q^{\alpha}$ with $\alpha \geq 4-\eta(d)$, where $\eta(d) \rightarrow 0$ as $d \rightarrow \infty$. Since the minimum gap between the rational numbers $r / q$ is $1 / Q^{2}$, there is a trivial bound for the number of solutions of (1.1):

$$
\left(\delta Q^{2}+1\right)(M+1),
$$

which gives the right order of magnitude for $\alpha \leq 2$. The potential applications have $\alpha$ in the range $2<\alpha<4$, which is still out of reach.

We allow $f(x)$ in (1.1) or $F(x)$ in (1.2) to have order of magnitude $\lambda \geq 1$. The number of possible rational numbers $r / q$ has order of magnitude $T=\lambda Q^{2}$; the parameters $M$ and $T$ in (1.1) are analogous to $M$ and $T$ in the book [3]. For $\lambda$ very large, we should allow $u(x)$ and $v(x)$ to have different degrees. We use the determinants

$$
D_{k, n}(f(x))=\operatorname{det}\left(\frac{f^{(k+i-j)}(x)}{(k+i-j) !}\right)_{n \times n},
$$

which are identically zero when $f(x)=u(x) / v(x)$ with the polynomials $u(x), v(x)$ of degrees at most $k-1$ and $n-1$ respectively.

We consider a $(2 d+2)$-tuplet of solutions of (1.1) or (1.2). If a determinant of order $2 d+2$ vanishes, then there is a curve $y=u(x) / v(x)$ through the $2 d+2$ points; we call this the major arc case. Other $(2 d+2)$-tuplets are called minor arcs. Our approach is in two stages, firstly simple ideas which require the evaluation of determinants, and secondly detailed study of the major arcs. This paper treats the first stage only. We use trivial arguments on the major arcs, and our results are non-trivial only when $\delta$ is zero or close to zero. In a subsequent paper we discuss the major arcs, and extend the range of $\delta$ a little, although not to all the ranges needed for applications.

TheOREM 1. Let $f(x)$ be a real function on $[0, M], 2 d+2$ times continuously differentiable, with

$$
\left|\frac{f^{(r)}(x)}{r !}\right| \leq \frac{\lambda C^{r+1}}{M^{r}}
$$


for $r=0, \ldots, 2 d+2$, and

$$
\left|D_{d+1, s}(f(x))\right| \geq\left(\frac{\lambda}{C^{d+2} M^{d+1}}\right)^{s}
$$

for $s=1, \ldots, d+1$. Let $S$ be a set of points $(m, r / q)$ with $m, r, q$ integers, $0 \leq m \leq M, 1 \leq q \leq Q,(r, q)=1$, satisfying

$$
\left|f(m)-\frac{r}{q}\right| \leq \frac{\Delta}{Q^{2}}
$$

where $\Delta<1 / 2, C \geq 1, M \geq 2, Q \geq 2$ and $\lambda$ are parameters with $T=$ $\lambda Q^{2} \geq 4$. Then the number of points in $S$ is at most

$$
A\left(C^{d+2} M^{d} T\right)^{1 /(2 d+1)}+A\left(C^{2 d^{3}+8 d^{2}+11 d+4} \Delta^{d+1} T^{d}\right)^{1 /\left(2(d+1)^{2}\right)} M,
$$

where $A$ is a constant depending only on $d$.

THEOREM 2. Let $F(x)$ be a real function on $[0,1], 2 d+2$ times continuously differentiable, with

$$
\left|\frac{F^{(r)}(x)}{r !}\right| \leq \lambda C^{r+1}
$$

for $r=0, \ldots, 2 d+2$, and

$$
\left|D_{d+1, s}(F(x))\right| \geq\left(\frac{\lambda}{C^{d+2}}\right)^{s}
$$

for $s=1, \ldots, d+1$. Let $S$ be a set of points $(m / n, r / q)$ with $m, n, r, q$ integers, $0 \leq m \leq n, 1 \leq n \leq M, 1 \leq q \leq Q,(m, n)=1,(r, q)=1$, satisfying

$$
\left|F\left(\frac{m}{n}\right)-\frac{r}{q}\right| \leq \frac{\Delta}{Q^{2}}
$$

where $\Delta<1 / 2, C \geq 1, M \geq 2, Q \geq 2$ and $\lambda$ are parameters with $T=$ $\lambda Q^{2} \geq 4$. Then the number of points in $S$ is at most

$$
A\left(C^{d+2} M^{2 d} T\right)^{1 /(2 d+1)}+A\left(C^{2 d^{3}+8 d^{2}+11 d+4} \Delta^{d+1} T^{d}\right)^{1 /\left(2(d+1)^{2}\right)} M^{2},
$$

where $A$ is a constant depending only on $d$.

In the applications to gaps between $k$-free numbers we have $f(x)=$ $\lambda(1+x / M)^{-s}$ in Theorem 1 or $F(x)=\lambda(1+x)^{-s}$ in Theorem 2 . For any $s \neq 0, \pm 1, \ldots, \pm d$ we can choose $C$ depending on $s$ so that the inequalities required are valid for $0 \leq x \leq 1$; we verify this in Section 4 .

2. The approximation determinant. Let $V\left(x_{1}, \ldots, x_{n}\right)$ denote the Vandermonde determinant

$$
V\left(x_{1}, \ldots, x_{n}\right)=\operatorname{det}\left(x_{i}^{j-1}\right)_{n \times n}=\prod_{r=1}^{n-1} \prod_{s=r+1}^{n}\left(x_{s}-x_{r}\right) .
$$


In this section we express more complicated determinants in terms of Vandermondians.

Lemma 2.1. Let $P=P_{r, s}\left(x_{1}, \ldots, x_{r+s}, y_{1}, \ldots, y_{r+s}\right)$ denote the determinant of size $r+s$ whose $i$-th row is

$$
\left(1, x_{i}, \ldots, x_{i}^{r-1}, y_{i}, x_{i} y_{i}, \ldots, x_{i}^{s-1} y_{i}\right) .
$$

Then $P$ is zero if and only if the $r+s$ points $\left(x_{i}, y_{i}\right)$ satisfy an equation

$$
y=u(x) / v(x),
$$

where $u(x), v(x)$ are polynomials of degree at most $r-1$ and $s-1$ respectively.

Proof. Writing the equation (2.2) as

$$
a_{r-1} x^{r-1}+\ldots+a_{0}=\left(b_{s-1} x^{s-1}+\ldots+b_{0}\right) y,
$$

we see that $P=0$ is the eliminant of the coefficients $a_{r-1}, \ldots, a_{0}, b_{s-1}, \ldots, b_{0}$.

LEMma 2.2. Let $F(x)$ be a real function, $r+s-1$ times continuously differentiable on an interval I of length $L$, whose derivatives satisfy

$$
\frac{\left|F^{(k)}(x)\right|}{k !} \leq \lambda C^{k+1}
$$

for $x$ on $I, k=0, \ldots, r+s-1$, where

$$
L \leq 1 / 2 C \leq 1 / 2 .
$$

Let $x_{1}, \ldots, x_{r+s}$ be distinct points in I. Then we have the following bounds. First

$$
\left|P_{r, s}\left(x_{1}, \ldots, x_{r+s}, F\left(x_{1}\right), \ldots, F\left(x_{r+s}\right)\right)\right| \leq E_{0} .
$$

If $y_{i}=F\left(x_{i}\right)+\delta_{i}$ with $\left|\delta_{i}\right| \leq \delta$, then

$$
\begin{gathered}
\mid P_{r, s}\left(x_{1}, \ldots, x_{r+s}, y_{1}, \ldots, y_{r+s}\right) \\
-P_{r, s}\left(x_{1}, \ldots, x_{r+s}, F\left(x_{1}\right), \ldots, F\left(x_{r+s}\right)\right) \mid \leq \sum_{t=1}^{s} \delta^{t} E_{t}, \\
\left|P_{r, s}\left(x_{1}, \ldots, x_{r+s}, y_{1}, \ldots, y_{r+s}\right)\right| \leq \sum_{t=0}^{s} \delta^{t} E_{t},
\end{gathered}
$$

where for $t=0, \ldots, s$

$$
\begin{aligned}
E_{t}=2^{s+t-1} s !(r+s-t)^{(r+s-t) / 2} C^{(r+1)(s-t)} & \\
& \times \lambda^{s-t}\left(\frac{L}{2}\right)^{\left((r+s-1)^{2}+(r+s-2 t)^{2}-1\right) / 4} .
\end{aligned}
$$

Proof. Let $x_{0}$ be the midpoint of $I$. The determinant is unchanged by linear shifts $x_{i} \rightarrow x_{i}-x_{0}$, so we may suppose that $x_{0}=0$, and $I$ is the interval $|x| \leq L / 2$. 
We expand $F(x)$ by its Taylor series $\sum c_{i} x^{i}$ about $x=0$, so

$$
\begin{aligned}
x_{i}^{v-1} F\left(x_{i}\right) & =c_{0} x_{i}^{v-1}+\ldots+c_{r+s-v-1} x_{i}^{r+s-2}+x_{i}^{r+s-1} \frac{F^{(r+s-v)}(\xi)}{(r+s-v) !} \\
& =\sum_{k=0}^{r+s-v} c(i, k, v) x_{i}^{k+v-1}
\end{aligned}
$$

for some $\xi=\xi_{i v}$. The coefficients $c(i, k, v)$ are the Taylor coefficients $c_{k}$ except when $k$ takes its maximum value $k=r+s-v$. They satisfy

$$
|c(i, k, v)| \leq C^{k+1} \lambda .
$$

We substitute (2.9) into column $r+v$ of $P$ for $v=1, \ldots, s$, and expand $P$ as a sum of further determinants $P(\mathbf{k})$ indexed by an $s$-tuple $\mathbf{k}=\left(k_{1}, \ldots, k_{s}\right)$ of integers, meaning that for $v=1, \ldots, s$, the $k_{v}$ th term in the expansion (2.9) is taken. If $v+k_{v} \leq r+s-1$, then the entry in row $i$, column $j=r+v$ is $c_{k} x^{v+k-1}$ for $k=k_{v}$. If $v+k_{v} \leq r$ for any $v$, or if any value of $v+k_{v}$ except $r+s$ occurs twice, then two columns are proportional, and the determinant indexed $\left(k_{1}, \ldots, k_{s}\right)$ is zero. For non-zero determinants, the values of $v+k_{v}$ for $v=1, \ldots, s$ are the integers $r$ to $r+s-1$, each at most once, or $r+s$, with repeats allowed. The number of vectors $\mathbf{k}$ for which $P(\mathbf{k}) \neq 0$ is

$$
\leq 2^{s-1} s !
$$

In a non-zero determinant $P(\mathbf{k})$, for each $g=r, \ldots, r+s-2$ there is at most one $v=v(g)$ with $v+k_{v}=g+1$, and the entries in column $v+r$ are $c_{g+1-v} x^{g}$. The other $v$ in $v=1, \ldots, s$ have $v+k_{v}=r+s$, and the entries in columns $v+r$ are $c(i, r+s-v, v) x^{r+s-1}$. We assign these $v$ arbitrarily to be the values of $v(g)$ for those $g$ in $r, \ldots, r+s-1$ for which $v(g)$ has not already been defined, in such a way that $v(g)$ is a one-to-one function from the set $\{r, \ldots, r+s-1\}$ to the set $\{1, \ldots, s\}$. Since $C L \leq 1$, in both cases the entries in column $g$ are bounded in absolute value by

$$
C^{g+2-v(g)} \lambda\left(\frac{L}{2}\right)^{g} .
$$

The absolute value of a determinant is bounded by the product of the lengths of the column vectors, so

$$
\begin{aligned}
|P(\mathbf{k})| & \leq(r+s)^{(r+s) / 2}\left(\prod_{u=1}^{r}\left(\frac{L}{2}\right)^{u-1}\right)\left(\prod_{g=r}^{r+s-1} C^{g+2-v(g)} \lambda\left(\frac{L}{2}\right)^{g}\right) \\
& =(r+s)^{(r+s) / 2}\left(\frac{L}{2}\right)^{(r+s)(r+s-1) / 2} \lambda^{s} \prod_{g+2=r+2}^{r+s-1} C^{g+2} \prod_{v=1}^{s} C^{-v} \\
& =(r+s)^{(r+s) / 2} C^{(r+1) s} \lambda^{s}\left(\frac{L}{2}\right)^{(r+s)(r+s-1) / 2} .
\end{aligned}
$$


We obtain the first result (2.5) of the lemma on multiplying (2.11) by the estimate (2.10) for the number of $\mathbf{k}$ for which $P(\mathbf{k})$ is non-zero.

For the result $(2.6)$ we expand $P_{r, s}\left(x_{1}, \ldots, x_{r+s}, y_{1}, \ldots, y_{r+s}\right)$ as a sum of $2^{r+s}$ determinants in which each row is either

$$
\left(1, x_{i}, \ldots, x_{i}^{r-1}, F\left(x_{i}\right), x_{i} F\left(x_{i}\right), \ldots, x_{i}^{s-1} F\left(x_{i}\right)\right)
$$

or

$$
\left(0,0, \ldots, 0, \delta_{i}, \delta_{i} x_{i}, \ldots, \delta_{i} x_{i}^{s-1}\right) .
$$

Consider a determinant in which the second choice has been made in $t$ rows. If $t>s$, then the determinant is zero. For $t \leq s$ we expand by the $t$ rows involving values of $\delta_{i}$. We must estimate $s ! /(s-t)$ ! minor determinants with rows of the form

$$
\left(1, x_{i}, \ldots, x_{i}^{r-1}, x_{i}^{v_{1}-1} F\left(x_{i}\right), x_{i}^{v_{2}-1} F\left(x_{i}\right), \ldots, x_{i}^{v_{s-t}-1} F\left(x_{i}\right)\right)
$$

with $1 \leq v_{1}<\ldots<v_{s-t} \leq s$. We follow the previous argument with $s$ replaced by $s-t$, writing

$$
x_{i}^{v-1} F\left(x_{i}\right)=\sum_{k=0}^{\max (r+s-t-v, 0)} c^{\prime}(i, k, v) x_{i}^{k+v-1} .
$$

Again $c^{\prime}(i, k, v)=c_{k}$ except when $k$ takes its maximum value, and

$$
\left|c^{\prime}(i, k, v)\right| \leq C^{k+1} \lambda \text {. }
$$

We expand into determinants $P(\mathbf{k})$ of order $r+s-t$ indexed by $(s-t)$-tuplets of integers $\left(k_{1}, \ldots, k_{s-t}\right)$, which are zero if $v_{j}+k_{j} \leq r$ for any $j$, or if any value of $v_{j}+k_{j}$ in the range $r+1, \ldots, r+s-t-1$ occurs twice, with $v_{j} \geq r+s-t$ forcing $k_{j}=0$. The number of non-zero determinants is at most $2^{s-t-1}(s-t)$ ! as in (2.10). As in (2.11) we have

$$
|P(\mathbf{k})| \leq(r+s-t)^{(r+s-t) / 2} C^{(r+1)(s-t)} \lambda^{s-t}\left(\frac{L}{2}\right)^{(r+s-t)(r+s-t-1) / 2} .
$$

So the terms in powers of $\delta$ are estimated by

$$
\begin{aligned}
& \sum_{t=1}^{s} \frac{s !}{(s-t) !} \delta^{t}\left(\frac{L}{2}\right)^{t(t-1) / 2} 2^{s-t-1}(s-t) ! \\
& \times(r+s-t)^{(r+s-t) / 2} C^{(r+1)(s-t)} \lambda^{s-t}\left(\frac{L}{2}\right)^{(r+s-t)(r+s-t-1) / 2} \\
& =\sum_{t=1}^{s} \delta^{t} E_{t}
\end{aligned}
$$

as required. 
Lemma 2.3. Let $F(x)$ be a real function, $r+s$ times continuously differentiable on an interval $I$ of length $L$, whose derivatives satisfy (2.3) for $k=0, \ldots, r+s$ with (2.4) holding. Let

$$
D_{r, s}(F(x))=\operatorname{det}\left(\frac{F^{(r+i-j)}(x)}{(r+i-j) !}\right)_{s \times s},
$$

a determinant of the Taylor coefficients of $F(x)$ about the point $x$; if $s>$ $r+1$, then we replace the undefined entries with $r+i-j<0$ by zero. Let $x_{1}, \ldots, x_{r+s}$ be distinct points in $I$, and let

$$
P=P_{r, s}\left(x_{1}, \ldots, x_{r+s}, F\left(x_{1}\right), \ldots, F\left(x_{r+s}\right)\right) .
$$

Then we have the estimate

$$
\begin{aligned}
& \left|P-D_{r, s}\left(F\left(x_{0}\right)\right) V\left(x_{1}, \ldots, x_{r+s}\right)\right| \\
\leq & 2^{r+2 s} s !(r+s-1)^{(r+s-1) / 2} C^{\left(2 r s+s^{2}+2 s+2\right) / 2} \lambda^{s}\left(\frac{L}{2}\right)^{\left((r+s)^{2}-r-s+2\right) / 2},
\end{aligned}
$$

where $x_{0}$ denotes the midpoint of $I$.

Proof. As in Lemma 2.2 we suppose that $I$ is the interval $[-L / 2, L / 2]$. Let

$$
g_{k}(x)=c_{0} x^{k-1}+c_{1} x^{k}+\ldots+c_{r+s-k} x^{r+s-1},
$$

in the notation of (2.9). Then

$$
x_{i}^{k-1} F\left(x_{i}\right)=g_{k}\left(x_{i}\right)+\eta_{i k},
$$

where

$$
\eta_{i k}=\frac{F^{(r+s+1-k)}\left(\xi_{i k}\right) x_{i}^{r+s}}{(r+s+1-k) !}
$$

for some $\xi_{i k}$ between 0 and $x_{i}$. We have

$$
\left|\eta_{i k}\right| \leq \eta_{k}=C^{r+s+2-k} \lambda\left(\frac{L}{2}\right)^{r+s}
$$

We expand the determinant $P_{r, s}\left(x_{1}, \ldots, x_{r+s}, F\left(x_{1}\right), \ldots, F\left(x_{r+s}\right)\right)$ as a sum of $2^{r+s}$ determinants in which each row is either

$$
\left(1, x_{i}, \ldots, x_{i}^{r-1}, g_{1}\left(x_{i}\right), g_{2}\left(x_{i}\right), \ldots, g_{s}\left(x_{i}\right)\right)
$$

or

$$
\left(0,0, \ldots, 0, \eta_{i 1}, \eta_{i 2}, \ldots, \eta_{i s}\right) .
$$

The first choice (2.15) for each row gives the determinant

$$
\operatorname{det}\left(\sum_{l=1}^{r+s} c_{l j} x_{i}^{l-1}\right)_{(r+s) \times(r+s)}=V \operatorname{det} C,
$$


where $V=V\left(x_{1}, \ldots, x_{r+s}\right)$, and $C$ is the matrix $\left(c_{i j}\right)$ with

$$
c_{i j}= \begin{cases}\delta_{i j} & \text { for } j \leq r, \\ c_{r+i-j} & \text { for } r+1 \leq j \leq r+i, \\ 0 & \text { for } r+i+1 \leq j \leq r+s\end{cases}
$$

The first $r$ columns of $C$ are those of an identity matrix, so the determinant of $C$ is equal to the determinant of its last $s$ rows and columns, which is $D_{r, s}(F(0))$ in the statement of the lemma (recall that the interval was shifted so that $\left.x_{0}=0\right)$.

For every other choice of rows we estimate the determinant as in Lemma 2.2. Consider a determinant where the choice (2.16) has been made in $t$ rows. If $t>s$, then the determinant is zero. If $t \leq s$, then we expand by these $t$ rows. We have to consider $s ! /(s-t)$ ! minor determinants with rows of the form

$$
\left(1, x_{i}, \ldots, x_{i}^{r-1}, g_{v_{1}}\left(x_{i}\right), g_{v_{2}}\left(x_{i}\right), \ldots, g_{v_{s-t}}\left(x_{i}\right)\right)
$$

with $1 \leq v_{1}<\ldots<v_{s-t} \leq s$. We write

$$
g_{v}\left(x_{i}\right)=\sum_{k=0}^{r+s-t-v} c^{\prime \prime}(i, k, v) x_{i}^{k+v-1},
$$

where $c^{\prime \prime}(i, k, v)=c_{k}$ except when $k$ takes its maximum value, when

$$
\begin{aligned}
c^{\prime \prime}(i, r+s-t & -v, v)=\frac{1}{(r+s-t-1) !} g_{v}^{(r+s-t-1)}(\xi) \\
& =\sum_{k=r+s-t-v}^{r+s-v} \frac{(k+v-1) !}{(r+s-t-1) !(k+v-r-s+t) !} c_{k} \xi^{k+v-r-s-t}
\end{aligned}
$$

for some $\xi=\xi_{i v}$ between 0 and $x_{i}$. Writing $u=r+s-t-1$, we have

$$
\begin{aligned}
\left|c^{\prime \prime}(i, u-v+1, v)\right| & \leq \sum_{j=0}^{t} \frac{(u+j) !}{j ! u !}\left|c_{u-v+j-1}\right|\left(\frac{L}{2}\right)^{j} \\
& \leq \lambda C^{u-v} \sum_{j=0}^{t} \frac{(u+j) !}{j ! u !}\left(\frac{C L}{2}\right)^{j} \leq \lambda C^{u-v}\left(1-\frac{C L}{2}\right)^{-u} \\
& \leq 2^{u} C^{u-v} \lambda,
\end{aligned}
$$

so that

$$
\left|c^{\prime \prime}(i, k, v)\right| \leq 2^{r+s-t-1} C^{k+1} \lambda .
$$

We expand into determinants $P(\mathbf{k})$ as in Lemma 2.2 ; the estimate for $P(\mathbf{k})$ 
in (2.12) must be multiplied by $2^{r+s-t-1}$. We note that

$$
\begin{aligned}
\max \eta_{k_{1}} \eta_{k_{2}} \ldots \eta_{k_{t}} & =\prod_{k=1}^{t} C^{r+s-r-k} \lambda\left(\frac{L}{2}\right)^{r+s} \\
& =C^{t(2 r+2 s-t+3) / 2} \lambda^{t}\left(\frac{L}{2}\right)^{(r+s) t},
\end{aligned}
$$

where the maximum is over sets of $t$ distinct integers taken from $1, \ldots, s$. So the terms with at least one factor $\eta_{i k}$ are estimated as

$$
\begin{aligned}
\sum_{t=1}^{s} & \frac{s !}{(s-t) !} C^{t(2 r+2 s-t+3) / 2} \lambda^{t}\left(\frac{L}{2}\right)^{(r+s) t} 2^{s-t}(s-t) ! \\
& \times 2^{r+s-t-1}(r+s-t)^{(r+s-t) / 2} C^{(r+1)(s-t)} \lambda^{s-t}\left(\frac{L}{2}\right)^{(r+s-t)(r+s-t-1) / 2} \\
\leq & \sum_{t=1}^{s} 2^{r+2 s-2 t-1} s !(r+s-1)^{(r+s-1) / 2} C^{r s+s t+s-t(t-1) / 2} \lambda^{s} \\
& \times\left(\frac{L}{2}\right)^{\left((2 r+2 s-1)^{2}+(2 t+1)^{2}-2\right) / 8} \\
\leq & 2^{r+2 s-1} s !(r+s-1)^{(r+s-1) / 2} C^{r s+s} \lambda^{s}\left(\frac{L}{2}\right)^{\left((r+s)^{2}-r-s\right) / 2} \\
& \times \sum_{t=1}^{s} C^{s t-t(t-1) / 2}\left(\frac{L}{2}\right)^{t(t+1) / 2} .
\end{aligned}
$$

The sum over $t$ is

$$
\sum_{t=1}^{s} C^{s t-t^{2}}\left(\frac{C L}{2}\right)^{t(t+1) / 2} \leq 2 \frac{C L}{2} C^{s^{2} / 2},
$$

which gives the result of the lemma.

3. Major and minor arcs. We discuss the local structure of the set $S$ of rational points $P_{i}\left(x_{i} y_{i}\right)$ in Theorems 1 and 2. For convenience we replace (1.1) by

$$
\left|F\left(\frac{m}{M}\right)-\frac{r}{q}\right| \leq \delta
$$

scaling the function $f(x)$ in (1.1) to satisfy the same conditions as in Theorem 2. After reducing $m / M$ to its lowest terms, we have $x_{i}=m_{i} / n_{i}$ with $n_{i} \mid M$; we call this Case 1 . Case 2 will denote $x_{i}=m_{i} / n_{i}$ with $1 \leq n_{i} \leq M$ as in Theorem 2. We number the points $P_{i}$ in order of $x_{i}$ increasing. 
A set of $2 d+2$ or more consecutive points of $S$ is called a major arc if the points satisfy an equation $y=u(x) / v(x)$ as in (2.2) with $u(x), v(x)$ polynomials of degree at most $d$. The points $P_{1}, \ldots, P_{2 d+2}$ lie on a major arc if and only if the determinant $P$ (with $r=s=d+1$ ) vanishes in Lemma 2.1. All other sets of $2 d+2$ consecutive points of $S$ are called minor arcs. For these, the determinant $P$ is a non-zero rational number, whose denominator is a factor of

$$
M^{d(d+1)} q_{1} q_{2} \ldots q_{2 d+2} \leq\left(M^{d} Q^{2}\right)^{d+1}
$$

in Case 1, and a factor of

$$
n_{1}^{d} n_{2}^{d} \ldots n_{2 d+2}^{d} q_{1} q_{2} \ldots q_{2 d+2} \leq\left(M^{d} Q\right)^{2 d+2}
$$

in Case 2. On a minor arc the numerator is numerically at least one, and we get a lower bound $L \geq L_{1}$ for the distance $L=x_{2 d+2}-x_{1}$.

Consider a block of $k \geq 2 d+2$ consecutive $P_{i}$ such that every consecutive $(2 d+2)$-tuplet forms a minor arc. There are $[(k-1) /(2 d+1)](2 d+2)$-tuplets with only endpoints in common, so the $k$ points occupy an interval of length

$$
L \geq\left[\frac{k-1}{2 d+1}\right] L_{1}
$$

and

$$
k \leq(2 d+1)\left(L / L_{1}+1\right)+1 \leq(4 d+3) L / L_{1} .
$$

These intervals are disjoint for different blocks of minor arc points, so the number of points of $S$ for which no consecutive $(2 d+2)$-tuplet containing them is a major arc is

$$
\leq(4 d+3) / L_{1}+2(2 d+1) \leq(4 d+3)\left(1 / L_{1}+1\right),
$$

where we have allowed for at most $2 d+1$ points left over at each end of the interval $[0,1]$.

We use Vinogradov's order of magnitude notation $E \ll F$ to mean that the expression $E$ satisfies $|E| \leq B F$ for some implied constant $B$ when the main parameters in $E$ are large. For example in the lemma below, the main parameters are $M$ (range of input) and $T$ (range of output), and the inequality holds uniformly for $M \geq A_{1}, T \geq A_{2}$ for some constants $A_{1}, A_{2}$.

Lemma 3.1. Suppose that $F(x)$ satisfies the conditions of Lemma 2.2 with $r=s=d+1$, and that the points $P_{1}, \ldots, P_{2 d+2}$ form a minor arc lying in an interval of length $L$. Then $L \geq L_{1}$, where

$$
\frac{1}{L_{1}} \ll \max _{0 \leq t \leq d+1}\left(\left(\frac{\delta}{\lambda}\right)^{t} C^{(d+2)(d+1-t)} \frac{T^{d+1}}{K^{d(d+1)}}\right)^{1 /\left(d^{2}+d+(d+1-t)^{2}\right)} .
$$

Here $T=\lambda Q^{2}, K=1 / M$ in Case $1, K=1 / M^{2}$ in Case 2, and the implied constants depend only on $d$. 
Proof. We use the lower bounds

$$
\left|P_{d+1, d+1}\right| \geq \frac{1}{\left(M^{d} Q^{2}\right)^{d+1}}
$$

in Case 1,

$$
\left|P_{d+1, d+1}\right| \geq \frac{1}{\left(M^{2 d} Q^{2}\right)^{d+1}}
$$

in Case 2, and we rearrange (2.7) of Lemma 2.2.

Lemma 3.1 is weaker than we would like. For large $\delta$, the degree $d$ should be close to $(\log Q) /(\log M)$, unless $\lambda$ is very large, when we should take $u(x)$, $v(x)$ of different degrees $r-1$ (close to $(\log \lambda Q) /(\log M))$ and $s-1$ (close to $(\log Q) /(\log M))$. The bounds $L \geq(2 d+1) / M^{2}$ in Case $2, L \geq(2 d+1) / M$ in Case 1 are trivial. When $t$ is close to $2 \sqrt{d}$, then Lemma 3.1 gives bounds worse than trivial unless $(\log \lambda / \delta) /(\log T)$ is approximately 2 or more. Our aim is bounds which are non-trivial as soon as $\delta<1 / Q^{2}, \log \lambda / \delta>\log T$.

If we consider points on the curve $(\delta=0)$, then there is a great simplification. We lose the terms in Lemma 3.1 with $t \geq 1$, and we can control the major arcs. In the case of $\delta$ small we can achieve this happy state by perturbing $F(x)$ with a function $g(x)$ which takes the values $\delta_{1}, \ldots, \delta_{2 d+2}$ at the points $x_{1}, \ldots, x_{2 d+2}$ :

$$
g(x)=\sum_{i=1}^{2 d+2} \delta_{i} \prod_{\substack{j=1 \\ j \neq i}}^{2 d+2} \frac{x-x_{j}}{x_{i}-x_{j}},
$$

a polynomial of degree $2 d+1$. Let

$$
L=x_{2 d+2}-x_{1}, \quad L_{0}=\min _{i \neq j}\left|x_{j}-x_{i}\right| .
$$

For $h=0, \ldots, 2 d+1$, the derivative $g^{(h)}(x)$ is a sum of $2 d+2$ times the binomial coefficient $2 d+1$ C $C_{h}$ terms, each with a product of $2 d+1-h$ factors $x-x_{i}$ on top, so for $x_{1} \leq x \leq x_{2 d+2}$,

$$
\left|g^{(h)}(x)\right| \leq \frac{(2 d+2)(2 d+1) !}{h !(2 d+1-h) !} \cdot \frac{\delta L^{2 d+1-h}}{L_{0}^{2 d}(L / 2)},
$$

where we have used the fact that for each $i$,

$$
\max _{j \neq i}\left|x_{j}-x_{i}\right| \geq L / 2 .
$$

We do not want to change the order of magnitude estimates for the determinants $P_{d+1, d+1}\left(x_{1}, \ldots, x_{2 d+2}, F\left(x_{1}\right), \ldots, F\left(x_{2 d+2}\right)\right)$ and $D_{r, s}(F(x))$ with $r=d+1,1 \leq s \leq d+1$, so we need a condition of the type

$$
\frac{\left|g^{(k)}(x)\right|}{k !} \leq \varepsilon \lambda C^{k+1}
$$


for $k=0, \ldots, 2 d+1$, with $\varepsilon \leq 1$. For the minor arcs argument in Lemma 2.2, we replace $F(x)$ by $F(x)+g(x)$ at the cost of replacing $C$ by $C+\varepsilon \leq C+1$. For the major arcs we write $D_{r, s}(F(x))$ as a sum of $2^{s}$ determinants, of which one is $D_{r, s}(F(x))$, and the other determinants have at least one row in which $F(x)$ is replaced by $g(x)$, and the upper estimates are smaller by a factor $\varepsilon$. The sum of the $2^{s}-1$ determinants involving values of $g(x)$ is in modulus

$$
\leq \varepsilon 2^{s} s ! C^{(r+1) s} \lambda^{s},
$$

which is at most one half the lower bound for $D_{r, s}(F(x))$ in Theorem 2 if

$$
\varepsilon \leq \frac{1}{2^{s+1} s ! C^{2(r+1) s}} .
$$

The worst case is $r=s=d+1$, when we require

$$
\varepsilon \leq \frac{1}{2^{d+2}(d+1) ! C^{2(d+1)(d+2)}} .
$$

This condition allows us to replace $F(x)$ by $F(x)+g(x)$ in Lemma 2.3 at a cost of replacing $C$ by $2^{1 /(d+1)} C$. The condition (3.6) is stronger than we need in Lemma 2.2, where we only use $\varepsilon \leq 1$ in (3.5). Since $C L<1,(3.4)$ is true for all $k \leq 2 d+1$ if

$$
\delta \leq \frac{\lambda L L_{0}^{2 d}}{2^{3 d+5}(d+2) ! C^{2(d+1)^{2}}} .
$$

Since a $(2 d+2)$-tuplet has $L \geq(2 d+1) L_{0}$, we have (3.7) for all $(2 d+2)$-tuplets of points of $S$, not necessarily consecutive, if

$$
\delta \leq \frac{(2 d+1) \lambda L_{0}^{2 d+1}}{2^{3 d+5}(d+2) ! C^{2(d+1)^{2}}} .
$$

This condition enables us to replace $F\left(x_{i}\right)$ by $F\left(x_{i}\right)+g\left(x_{i}\right)=y_{i}$ at the cost of replacing $C$ by $2 C$.

For our next lemma we quote Theorem 2 of [6]:

Lemma 3.2. Let $f(x)$ be a real function, $2 d+1$ times continuously differentiable on an interval $I$. For $1 \leq r \leq n, n+r \leq 2 d+2$, let

$$
B_{r}(n, x)=\operatorname{det}\left(\frac{f^{(n+i-j)}(x)}{(n+i-j) !}\right)_{r \times r} .
$$

Suppose that for each $r=1, \ldots, d+1$, the function $B_{r}(d+1, x)$ does not vanish on $I$. Then if $u(x), v(x)$ are any polynomials of degrees at most $d$, the equation $f(x)=u(x) / v(x)$ has at most $2 d+1$ distinct roots in $I$, and for $\delta>0$, the points of I which satisfy

$$
\left|f(x)-\frac{u(x)}{v(x)}\right| \leq \delta
$$

form at most $2 d+2$ disjoint subintervals of $I$. 
The determinants in Lemma 3.2 are

$$
B_{r}(d+1, x)=D_{d+1, r}(F(x))
$$

in our present notation, and the conclusion is that no $(2 d+2)$-tuplet of points of $S$, whether consecutive or not, can lie on a rational curve $y=u(x) / v(x)$. Hence the determinant $P\left(x_{1}, \ldots, x_{2 d+2}, F\left(x_{1}\right), \ldots, F\left(x_{2 d+2}\right)\right)$ is non-zero.

If $\delta$ is so small that (3.8) holds with $L_{0}=1 / M$ in Case $1,1 / M^{2}$ in Case 2, then for each $(2 d+2)$-tuplet of consecutive points of $S$, we replace $F(x)$ by $F(x)+g(x)$ in Lemmas 3.1 and 3.2. The conditions on the derivatives in Lemmas 2.2, 2.3 and 3.2 hold with $C$ replaced by $2 C$. Lemma 3.2 says that the $(2 d+2)$-tuplet cannot be a major arc. Hence (3.2) gives a bound for the number of points of $S$, with $L_{1}$ given by the term $t=0$ in (3.3) of Lemma 3.1, as

$$
\ll\left(\frac{C^{d+2} T}{K^{d}}\right)^{1 /(2 d+1)} .
$$

If (3.8) does not hold, then we choose a prime $p$ (in Case 2), and we divide $S$ into $p+1$ parts. The set $S_{\infty}$ contains all points with $p \mid n_{i}$. For $c=0, \ldots, p-1$ the set $S_{c}$ contains all points with $m_{i} \equiv c n_{i}(\bmod p)$. If $\left(x_{i}, y_{i}\right)$ and $\left(x_{j}, y_{j}\right)$ lie in the same set $S_{k}$, then

$$
x_{j}-x_{i}=\frac{m_{j}}{n_{j}}-\frac{m_{i}}{n_{i}}=\frac{h}{n_{i} n_{j}}
$$

with $p \mid h$. Hence

$$
\left|x_{j}-x_{i}\right| \geq L_{0}=p / M^{2} .
$$

We take the prime $p$ so large that (3.8) holds with this value of $L_{0}$. For $c=0, \ldots, p-1$ we shift the values of $x$ in the points of $S_{c}$ by $c$, replacing $m_{i} / n_{i}$ by $k_{i} / n_{i}$ with $k_{i}=m_{i}-c n_{i}$; this shift does not affect the estimates for the derivatives of $f(x)$. The determinant $P$ can be written as

$$
\frac{Q}{n_{1}^{d} \ldots n_{2 d+2}^{d} q_{1} \ldots q_{2 d+2}}
$$

where $Q$ is the determinant whose $i$ th row is

$$
\left(n_{i}^{d} q_{i}, k_{i} n_{i}^{d-1} q_{i}, \ldots, k_{i}^{d} q_{i}, n_{i}^{d} r_{i}, k_{i} n_{i}^{d-1} r_{i}, \ldots, k_{i}^{d} r_{i}\right) ;
$$

for $S_{\infty}$ we have $m_{i}$ in place of $k_{i}$. We see that $Q$ is an integer divisible by $p^{d(d+1)}$.

In Case 1 we pick the prime $p$ at the start of the argument, when the points of $S$ are written as $\left(m_{i}, r_{i} / q_{i}\right)$, and divide into classes according to $m_{i} \equiv c(\bmod p)$, and in the class $S_{c}$, shift from $m_{i}$ to $l_{i}=m_{i}-c$. In the class $S_{c}$, when we replace $l_{i}$ by $k_{i} / n_{i}$, the lowest terms form of $l_{i} / M$, we have either $n_{i} \mid M / p$ for all $i$ or $p \mid l_{i}$ for all $i$. In both cases the spacing of 
points is at least $L_{0}=p / M$, and the determinant is

$$
P=\frac{Q}{M^{d(d+1)} q_{1} \ldots q_{2 d+2}},
$$

where $Q$ is again an integer divisible by $p^{d(d+1)}$.

The argument of Lemma 3.1 gives (3.9) with $K=L_{0}$. The total number of points of $S$ is thus in Case 2

$$
\ll\left(C^{d+2} p^{d+1} M^{2 d} T\right)^{1 /(2 d+1)},
$$

which becomes

$$
\ll\left(C^{2 d^{3}+8 d^{2}+11 d+4} \Delta^{d+1} T^{d}\right)^{1 /\left(2(d+1)^{2}\right)} M^{2}
$$

when we choose the prime $p$ so that the two sides of (3.8) have the same order of magnitude. In Case 1 we obtain the bound (3.10) with $M$ in place of $M^{2}$. This completes the proof of Theorems 1 and 2 .

4. Determinants formed with power functions. In many applications in number theory, the function $F(x)$ or $f(x)$ takes the form $N x^{-s}$ on $1 / 2 \leq x \leq 1$, or, after a change of variable, $\lambda(1+x)^{-s}$ on $0 \leq x \leq 1$. We calculate the determinant $D_{k, n}\left(\lambda(1+x)^{-s}\right)$ explicitly, and verify that it is non-zero except when $F(x)=u(x) / v(x)$ with $u(x)$ of degree at most $k-1$, $v(x)$ of degree at most $n-1$, that is, when $s$ is an integer with $0 \leq s \leq n-1$ or $1-k \leq s \leq 0$. We have

$$
\begin{aligned}
& D_{k, n}\left(\lambda(1+x)^{-s}\right)=\operatorname{det}\left(\frac{(-1)^{k+i-j} s(s+1) \ldots(s+k+i-j-1) \lambda}{(k+i-j) !(1+x)^{s+k+i-j}}\right)_{n \times n} \\
&=(-1)^{n k} \lambda^{n}\left(\prod_{i=1}^{n} \frac{s(s+1) \ldots(s+k+i-n-1)}{(k+i-n) !(1+x)^{s+k+i}}\right) \\
& \quad \times \operatorname{det}\left(\frac{(-1)^{j}(s+k+i-n) \ldots(s+k+i-j-1)(1+x)^{j}}{(k+i-n+1) \ldots(k+i-j)}\right)_{n \times n} \\
&=\frac{(-1)^{n k+n(n+1) / 2} s^{n}(s+1)^{n} \ldots(s+k-n)^{n}}{(k+1-n) !(k+2-n) ! \ldots k !} \\
& \quad \times \frac{(s+k-n+1)^{n-1} \ldots(s+k-1) \lambda^{n}(-(1+x))^{n(n+1) / 2} K}{(1+x)^{n(k+s)+n(n+1) / 2}} \\
&= \frac{(-1)^{n k} s^{n}(s+1)^{n} \ldots(s+k-n)^{n}(s+k-n+1)^{n-1} \ldots(s+k-1) \lambda^{n} K}{(k+1-n) !(k+2-n) ! \ldots k !(1+x)^{n(k+s)}},
\end{aligned}
$$

where $K$ is the numerical determinant

$$
K=\operatorname{det}\left(\frac{(s+k+i-n) \ldots(s+k+i-j-1)}{(1+k+i-n) \ldots(k+i-j)}\right)_{n \times n}=K_{n}(k+s, k+1),
$$


where

$$
K_{n}(s, t)=\operatorname{det}\left(\frac{(s+i-n) \ldots(s+i-j-1)}{(t+i-n) \ldots(t+i-j-1)}\right)_{n \times n} .
$$

In the evaluation of $K_{n}(s, t)$ we use the notation $t$, appropriate when $t$ is an integer. The method works for $t$ not an integer if we interpret the factorials as ratios of gamma functions. We subtract each row $i$ from the following row $i+1$, noting that

$$
\begin{gathered}
\frac{(s+i+1-n) \ldots(s+i-j)}{(t+i+1-n) \ldots(t+i-j)}-\frac{(s+i-n) \ldots(s+i-j-1)}{(t+i-n) \ldots(t+i-j-1)} \\
=\frac{(n-j)(t-s)(s+i+1-n) \ldots(s+i-j-1)}{(t+i-n) \ldots(t+i-j)} .
\end{gathered}
$$

In particular, after this subtraction we have $n-1$ zeros in rows $i=2$ to $n$ of column $j=n$. Expanding by the last column and renumbering rows 2 to $n$ as rows 1 to $n-1$, we have

$$
\begin{aligned}
& K_{n}(s, t) \\
& =(-1)^{n-1} \operatorname{det}\left(\frac{(n-j)(t-s)(s+i+1-n) \ldots(s+i-j-1)}{(t+i-n) \ldots(t+i-j)}\right)_{(n-1) \times(n-1)} \\
& =(s-t)^{n-1}(n-1) !\left(\prod_{i=1}^{n-1} \frac{1}{(t+i-n)(t+i-1-n)}\right) K_{n-1}(s, t+1) \\
& =(s-t)^{n-1}(n-1) ! \frac{(t-n) !(t-n+1) !}{(t-1) ! t !} K_{n-1}(s, t+1) .
\end{aligned}
$$

Iterating this process gives

$$
\begin{aligned}
& K_{n}(s, t) \\
& =\frac{(s-t)^{n-1}(s-t-1)^{n-2} \ldots(s-t-n+2) 2 ! \ldots(n-1) !(t-n) ! \ldots(t+n-3) !}{(t-1) ! t !^{2}(t+1) !^{2} \ldots(t+n-3) !^{2}(t+n-2) !} \\
& =\frac{(s-t)^{n-1}(s-t-1)^{n-2} \ldots(s-t-n+2) 2 ! \ldots(n-1) !(t-n) ! \ldots(t-2) !}{t !(t+1) ! \ldots(t+n-2) !} .
\end{aligned}
$$

Hence

$$
\begin{aligned}
D_{k, n}\left(\lambda(1+x)^{-s}\right) & =\frac{(-1)^{n k}(s-n+1)(s-n+2)^{2} \ldots(s-1)^{n-1} s^{n}}{k !(k-1) ! \ldots} \\
& \times \frac{(s+1)^{n} \ldots(s+k-n)^{n}(s+k-n-1)^{n-1} \ldots(s+k-1) \lambda^{n}}{(k-n+1) !(1+x)^{n(k+s)}},
\end{aligned}
$$

which is non-zero unless $s=-k+1,-k+2, \ldots, n-1$, and in these cases the determinant is identically zero. In all other cases the absolute value of the determinant is bounded below on $1 \leq x \leq 2$, and for $C$ sufficiently large the lower bounds required in Theorem 2 are valid for $k=d+1, n=1, \ldots, d+1$. 


\section{References}

[1] M. Filaseta and O. Trifonov, The distribution of fractional parts with applications to gap results in number theory, Proc. London Math. Soc. (3) 73 (1996), 241-278.

[2] M. N. Huxley, The rational points close to a curve, Ann. Scuola Norm. Sup. Pisa Cl. Sci. (4) 21 (1994), 357-375.

[3] —, Area, Lattice Points and Exponential Sums, Oxford Univ. Press, 1996.

[4] - Moments of differences between square-free numbers, in: Sieve Methods, Exponential Sums and their Applications in Number Theory, G. R. H. Greaves, G. Harman and M. N. Huxley (eds.), Cambridge Univ. Press, 1996, 187-204.

[5] —, The rational points close to a curve II, Acta Arith. 93 (2000), 201-219.

[6] - A determinant mean value theorem, to appear.

[7] M. N. Huxley et P. Sargos, Points entiers au voisinage d'une courbe plane de classe $C^{n}$, Acta Arith. 69 (1995), 359-366.

[8] V. Jarník, Über die Gitterpunkte auf konvexen Kurven, Math. Z. 24 (1925), 500-518.

[9] H. P. F. Swinnerton-Dyer, The number of lattice points on a convex curve, J. Number Theory 6 (1974), 128-135.

School of Mathematics

University of Cardiff

23 Senghenydd Road

Cardiff CF24 4YH

Wales, Great Britain

E-mail: huxley@cardiff.ac.uk

Received on 5.2.2002

and in revised form on 6.3.2003 